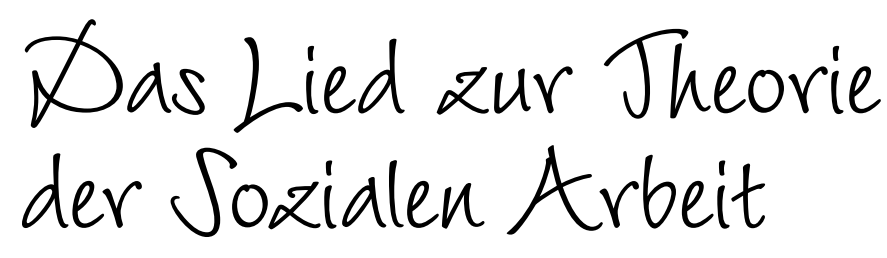

\title{
Eine Entstehungsgeschichte
}

Wie entsteht ein Lied zur Theorie der Sozialen Arbeit? Im Masterstudiengang soll man lernen, Soziale Arbeit zu entwickeln und zu gestalten, innovativ zu sein und Neues in die Welt zu bringen.

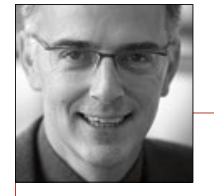

Roman UIram *1969

Gesang, Gitarre, Bass, Komposition seit 1984; Diplom an der Bundesakademie für Sozialarbeit in St. Pölten 1992. Masterstudierender an der FH Feldkirchen im Studiengang Soziale Arbeit. Musiker bei DRY acoustic (www.dry-acoustic.at). RomanHermann. UIram@edu.fhkaernten.ac.at

Dem Anspruch nach Wissenschaftlichkeit folgend ist es notwendig, den Gegenstand „Soziale Arbeit“ benennen zu lernen. Und die Theorien, die die Soziale Arbeit beschreiben, zu verstehen. Diese Themen haben wir uns vorgenommen, im Rahmen der Buchpräsentation „Theorie Sozialer Arbeit verstehen“ an der FH anschaulich zu erklären. Gemeinsam entwickelten wir Ideen, leicht verständliche Zugänge zur Theorie zu eröffnen. Unser Beitrag fand schließlich unter dem Titel „Vom Hammer zur Theorie Sozialer Arbeit" Eingang in das offizielle Programm der Buchvorstellung. Im Zuge der Ideenfindung fiel der Vorschlag, ein bekanntes Lied zu adaptieren. Die Fähigkeit zur Veränderung der Perspektive ist eine wesentliche Voraussetzung zum Verstehen. Und einen besonders guten Überblick hat man von oben. Über den Wolken habe ich einen strategisch günstigen Ort ausgemacht. Bereits Reinhard Mey wies darauf hin, dass Freiheit von Ängsten und Sorgen durch Distanzierung und Vergrößerung der Entfernung zu erlangen ist. Diese Perspektive verstand ich als Empfehlung. Als Handlungsanleitung zum Veranschaulichen des Verstehens der Theorie Sozialer Arbeit. Es schien mir angemessen, neben Otger Autrata und Bringfriede Scheu auch Hans Thiersch, Lothar Böhnisch, Silvia Staub-Bernasconi, Martha Nussbaum, Wolfgang Hinte und Pierre Bourdieu zu Wort zu bitten.

„Das Lied zur Theorie der Sozialen Arbeit“ bei youtube: https://www.youtube.com/watch?v=E3Kvn8TGiLc

\section{Das Lied zur Theorie der Sozialen Arbeit}

1) An der FH, Hörsaal null-drei, bis hier hör 'ich die Professoren.

All die Theorien zieh'n vorbei, und es dröhnt in meinen Ohren. Grau ist alle Theorie, sie quillt und staubt aus allen Poren. So was Trock'nes check' ich nie ich bin total verloren.

Chorus)

Über den Wolken

muss Verstehen wohl grenzenlos sein. Alle Rätsel, alle Fragen, sagt man, überblickt man ganz genau, und dann würde was uns unverständlich erscheint, plötzlich einfach und klar.

2) Ob Lothar Schön is? Mich Martert Nussbaum,

Hans Thiersch Staubt wie Bernasconi.

Hinte is vorn im Sozialraum,

ich trink Bourdieu zu Rigatoni.

Meine Gedanken haben schon

jede Orientierung verloren,

denn von fern klingen monoton

die Theorien der Professoren.

3) Autrata schreibt ein neues Buch,

ich Scheu mich nicht es zu lesen.

Selbst Sisyphus versucht

den Sinn zu verstehen.

Ich hab Erkenntnis angestrebt,

doch Sisyphus, der steht im Regen,

bis er abhebt und schwebt

der Praxis entgegen.

Musik: Reinhard Mey

Text: Roman Ulram 